DOI: https://doi.org/10.46296/ig.v3i5.0011

\title{
DESARROLLO E IMPLEMENTACIÓN DEL SISTEMA DE GESTIÓN EDITORIAL DE LA ULEAM
}

\section{DEVELOPMENT AND IMPLEMENTATION OF THE EDITORIAL MANAGEMENT SYSTEM OF ULEAM}

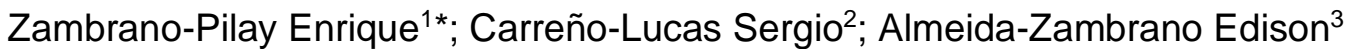 \\ 1 Universidad Laica Eloy Alfaro de Manabí, ULEAM. Manta, Ecuador. \\ ${ }^{2}$ Universidad Laica Eloy Alfaro de Manabí, ULEAM. Manta, Ecuador. \\ ${ }^{2}$ Profesor de la Universidad Laica Eloy Alfaro de Manabí, ULEAM. Manta, Ecuador.
}

*Correo: e1315391167@live.uleam.edu.ec

\begin{abstract}
Resumen
La complejidad de los sistemas de educación moderno exige el desarrollo de mecanismos que permitan potencializar las actividades de la difusión científica. Es por esta razón que la Editorial Universitaria de la Universidad Laica Eloy Alfaro de Manabí se ve en la necesidad de poseer un sistema que permita administrar la gestión de publicaciones para sistematizar el proceso de manera armónica entre autores, revisores y lectores que transmiten ese aporte epistemológico que facilita e intercambia el desarrollo del contenido científico. El sistema editorial de gestión universitaria de publicaciones es una plataforma digital que facilita la comunicación desde los diferentes escenarios sin la necesidad demográfica de contactarse de manera física con la editorial. En base a las necesidades detectadas en el departamento en mención y después de realizar un análisis de los puntos críticos es necesario realizar las contingencias que permitan validar la información pertinente con rigor científico.
\end{abstract}

Palabras clave: web, sistema, edición, publicación, plataforma.

\begin{abstract}
The complexity of modern education systems requires the development of mechanisms that allow the activities of scientific dissemination to be potentiated. It is for this reason that the University Editorial of the Universidad Laica Eloy Alfaro de Manabí sees the need to have a system that allows managing the publication management to systematize the process in a harmonious way among authors, reviewers and readers who transmit this epistemological contribution that facilitates and exchanges the development of scientific content. The publishing publishing university management system is a digital platform that facilitates communication from different scenarios without the demographic need to physically contact the publisher. Based on the needs detected in the mentioned department and after carrying out an analysis of the critical points, it is necessary to carry out contingencies that allow validating the relevant information with scientific rigor.
\end{abstract}

Keywords: web, system, edition, publication, platform.

Información del manuscrito:

Fecha de recepción: 18 de noviembre de 2019

Fecha de aceptación: 23 de diciembre de 2019

Fecha de publicación: 10 de enero de 2020 


\section{Introducción}

En 2016 Artola \& Gaitán realizaron el trabajo sobre "desarrollo e implementación del portal de la revista Científica "RCIEN" de la facultad de Ciencias e Ingenierías de la UNAN-Managua", donde se detalla la implementación de una revista científica con fines educativos, mediante desarrolladores web como: iconmon, jquery, loremipsun, lorempixel y HTML5. De esta forma se puede brindar a los usuarios una herramienta virtual que permita la síntesis y acceso a la información relacionada a las noticias tanto nacionales, internaciones en los ámbitos de tecnología, ciencia y estudios.

En 2013 Mamaui presentó el trabajo "Implementación del sistema de gestión de proyectos de investigación (G Project)", que detalla los aspectos más relevantes de la implementación de un sistema para mitigar los problemas de la gestión de trabajos y/o proyectos de investigación en el departamento de DICYX, compuesto por un entorno que ofrecía la posibilidad de proponer una solución informática que ayude en la gestión de la información

referente

a

investigaciones y proyectos de una forma más rápida y eficiente.

Por lo cual, se realizó el proceso de desarrollo e implementación del sistema editorial de gestión universitaria de publicaciones para la Editorial Universitaria de la ULEAM, con el fin de agilizar los procesos que son llevados a cabo en este departamento; se inicia la puesta en marcha del sistema para poder comprobar el diseño propuesto, obteniendo los principales requerimientos que los usuarios necesitan en el sistema a implementar, llevado a cabo mediante la utilización de la metodología ágil SCRUM, en la cual podemos identificar roles, productos a entregar, diseño, codificación, pruebas e implementación; además de poder identificar las especificaciones técnicas, objetivos, determinación de recursos que se necesitarán en el proceso de desarrollo.

Por lo mencionado anteriormente, en este trabajo se detalla la implementación y funcionamiento del sistema y además se indica la finalidad del mismo; así como otros escenarios que se tomarán en 
cuenta, tales como el lenguaje de programación y metodología de trabajo.

\section{Metodología}

Se aplicó la metodología de trabajo SCRUM en el diseño y desarrollo del Sistema Editorial de Gestión Universitaria de Publicaciones de la Editorial Uleam. A lo largo del proceso se incluye el ciclo de vida del producto backlog, adquisición y suministro de información.

\subsection{Diseño}

El presente trabajo de investigación se basa en el desarrollo de una aplicación/sistema web que lleve a cabo los procesos de edición, revisión y publicación de libros y revistas que se produce en la Editorial de la ULEAM. Especificando los procesos y mecanismos necesarios que la Editorial requiere, para el proceso de desarrollo e implementación de esta plataforma se utilizaron las siguientes herramientas:

- Lenguaje de programación.

- Servidor.

- Bases de datos.

- Entorno de desarrollo.

\subsection{Servicio Web}

Jorge (2016) indica que "un servicio web (en inglés, Web Service o Web services) es una tecnología que utiliza un conjunto de protocolos y estándares que sirven para intercambiar datos entre aplicaciones." También se pueden catalogar como aplicaciones a las cuales se puede llegar a través de un enlace o "link", el cual contendrá la información que esta resguardada en el servicio web para su posterior utilización o visualización.

\subsection{PHP}

Fumas (2014) indica que "PHP (Hypertext Preprocessor) es un lenguaje de programación interpretado que se utiliza para la generación de páginas web de forma dinámica". Este lenguaje de programación es catalogado como un lenguaje asociado al servidor, el cual ofrece diferentes ventajas:

- Multiplataforma.

- Fácil de aprender.

- Lenguaje modular.

- Programación orientada a objetos.

- Lenguaje de código libre y gratuito.

- Múltiples framework. 
- Buena integración con gestores de base de datos.

\subsection{Especificaciones técnicas}

\subsubsection{XAMPP}

Zepeda (2015) indica que "XAMPP es un paquete formado por un servidor web Apache, una base de datos MySQL y los intérpretes para los lenguajes PHP y Perl". Posee licencia GNU GPL y por esto, se convierte en un elemento muy importante en el desarrollo de páginas y sistemas web. Cabe aclarar que el nombre "XAMPP" viene de: $X$ (cualquier sistema operativo), A (Apache), M (MySQL), $\mathrm{P}$ (PHP) y $\mathrm{P}$ (Perl).

\subsubsection{MySQL}

MySQL es un sistema de gestión de base de datos relacional (RDBMS) de código abierto, basado en lenguaje de consulta estructurado (SQL). Además, se asocia con las aplicaciones basadas en la web y la publicación en línea y es un componente importante de una pila empresarial de código abierto llamado LAMP, siendo una plataforma de desarrollo web que utiliza Linux como sistema operativo, Apache como servidor web, MySQL como sistema de gestión de base de datos relacional y PHP como lenguaje de programación orientado a objetos (Rouse, 2015).

\subsubsection{Visual Studio Code}

Visual Studio Code es un editor de código fuente potente y ligero, el cual se ejecuta en el ordenador, y está disponible para las plataformas: Windows, MacOS y Linux. Viene con soporte incorporado para JavaScript, TypeScript y Node.js y tiene un rico ecosistema de extensiones para otros lenguajes $(\mathrm{C}++, \mathrm{C} \#$, Java, Python, PHP, Go) y tiempos de ejecución (.NET y Unity). (Microsoft, 2019).

\subsubsection{Apache}

Gustavo (2019) indica que "Apache HTTP Server es un software de servidor web gratuito y de código abierto para plataformas Unix con el cual se ejecutan el $46 \%$ de los sitios web de todo el mundo. Es mantenido y desarrollado por la Apache Software Foundation".

\subsubsection{HTML}

Diego (2012) indica que "HTML está a cargo de la estructura del documento y provee un grupo completo de elementos para este propósito. La especificación también 
incluye algunos elementos con la única tarea de proveer estilos".

\subsubsection{HTML 5}

HTML 5 es la última versión de HTML, el cual conlleva 2 conceptos diferentes; al tratarse de una nueva versión de HTML cuenta con nuevos elementos, atributos y comportamientos. Permite que las aplicaciones y los sitios web tomen un rumbo nuevo al alcanzar las mismas un punto más alto en diversidad (Mozilla, 2019b).

\subsubsection{CSS}

Mozilla (2019a) indicó que las "hojas de estilo en cascada (Cascading Style Sheets o CSS) corresponden al lenguaje utilizado para describir la presentación de documentos HTML o XML, esto incluye varios lenguajes basados en XML como son XHTML o SVG. CSS describe como debe ser renderizado el elemento estructurado en pantalla, en papel, hablado o en otros medios". Es por esto que, fue utilizado en el desarrollo del sistema propuesto, ya que funciona en conjunto con HTML, y además proporciona la estructura del contenido y el CSS generaría el diseño que se presentó en la fase final.

\subsubsection{JavaScript}

Según Flanagan (2017) JavaScript es considerado el lenguaje interpretado más usado en el diseño y desarrollo de sitios y páginas web; está conformado por una sintaxis similar a Java y C. Técnicamente, JavaScript es un lenguaje de programación interpretado, es decir que no se necesita compilar otro programa para ponerlo en funcionamiento, gracias a esto se puede observar directamente en cualquier explorador sin la necesidad de involucrar más procesos.

\subsubsection{SCRUM}

Se entiende por SCRUM a un método de trabajo en equipo en el cual se especifican roles para la gestión de proyectos involucrados con el desarrollo de Software, se basa en 3 pilares, los cuales son: transparencia, la cual asegura la visibilidad de los procesos en aquellos aspectos que pueden ocasionar inconvenientes en los resultados; la inspección, es la que ayuda en la detección de cambios indeseados en el proceso y la adaptación, nos conlleva a realizar cambios pertinentes para minimizar los impactos de los mismos. (Fernández-Martínez et al., 2013). 


\section{Resultados y discusión}

Un punto importante para las personas que intervienen en el proceso de edición, revisión y publicación de textos, según el proceso llevado a cabo en la Editorial Universitaria, es la agilidad y facilidad de manejo, es por esto que, la herramienta debe desarrollarse teniendo en cuenta la agilidad y la interacción que tendrán los involucrados con esta herramienta.

La aplicación de las herramientas de recolección de información consigue una mejor y mayor comprensión de la realidad del problema presente en la Editorial Universitaria, de esta forma se plantea de forma más acertada una propuesta dirigida a las necesidades detectadas en la Editorial, esta información es de vital importancia y da paso a la recolección de requisitos. $Y$ además permite realizar la implementación de una herramienta tecnológica que facilita los procesos de edición, revisión y publicación de libros y revistas científicas, lo cual será de ayuda para los actores que intervienen en dichos procesos, ya que brindará mayor agilidad, seguridad y orden de los procesos ya mencionados.

\subsection{Arquitectura}

A continuación, se mostrará la arquitectura del sistema, evidenciando el resultado de agrupar diversos elementos informáticos que serán tomados en cuenta para obtener un mayor rendimiento en el funcionamiento y desempeño del sistema.

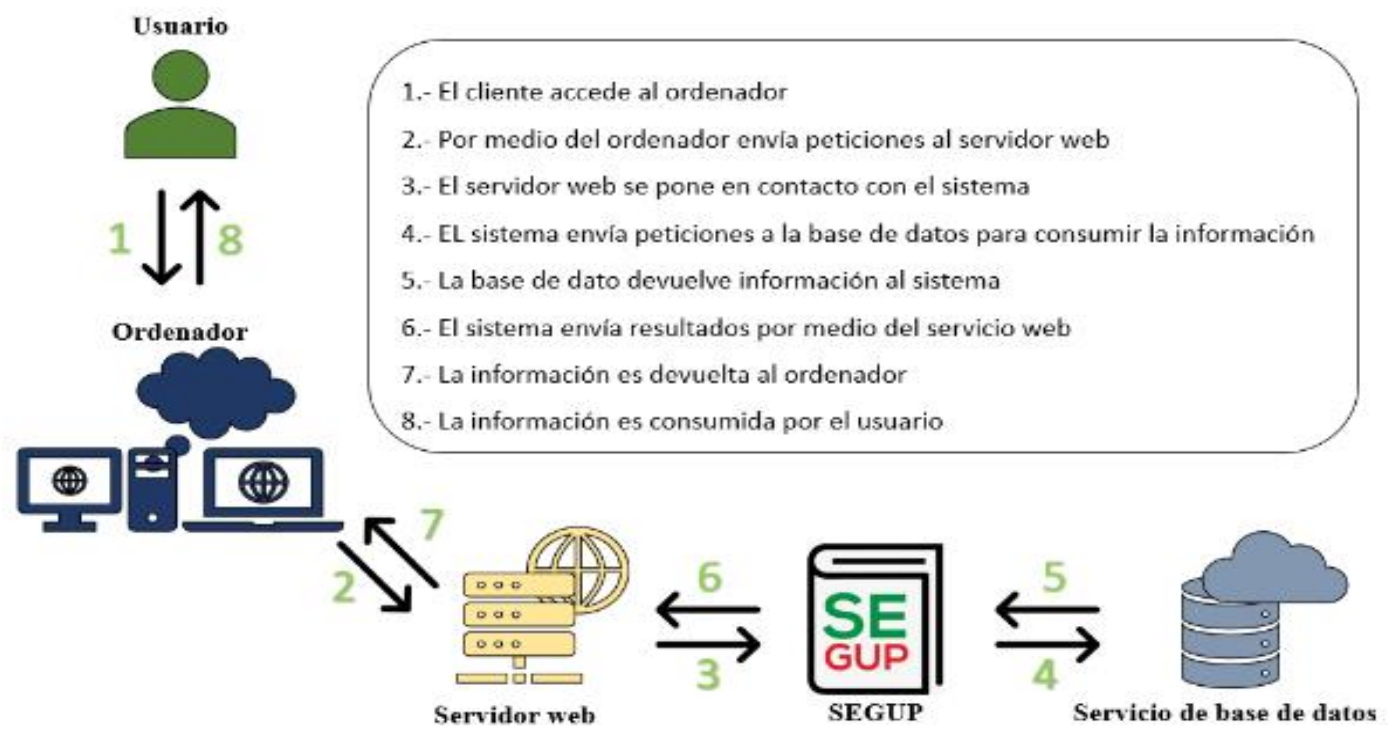

Figura 1. Arquitectura del sistema 


\subsection{Modelo de base de datos}

En este apartado se mostrará un diagrama representativo de la base de datos con todas sus relaciones y limitaciones; cabe señalar que estos aspectos aportan en el desarrollo del sistema.

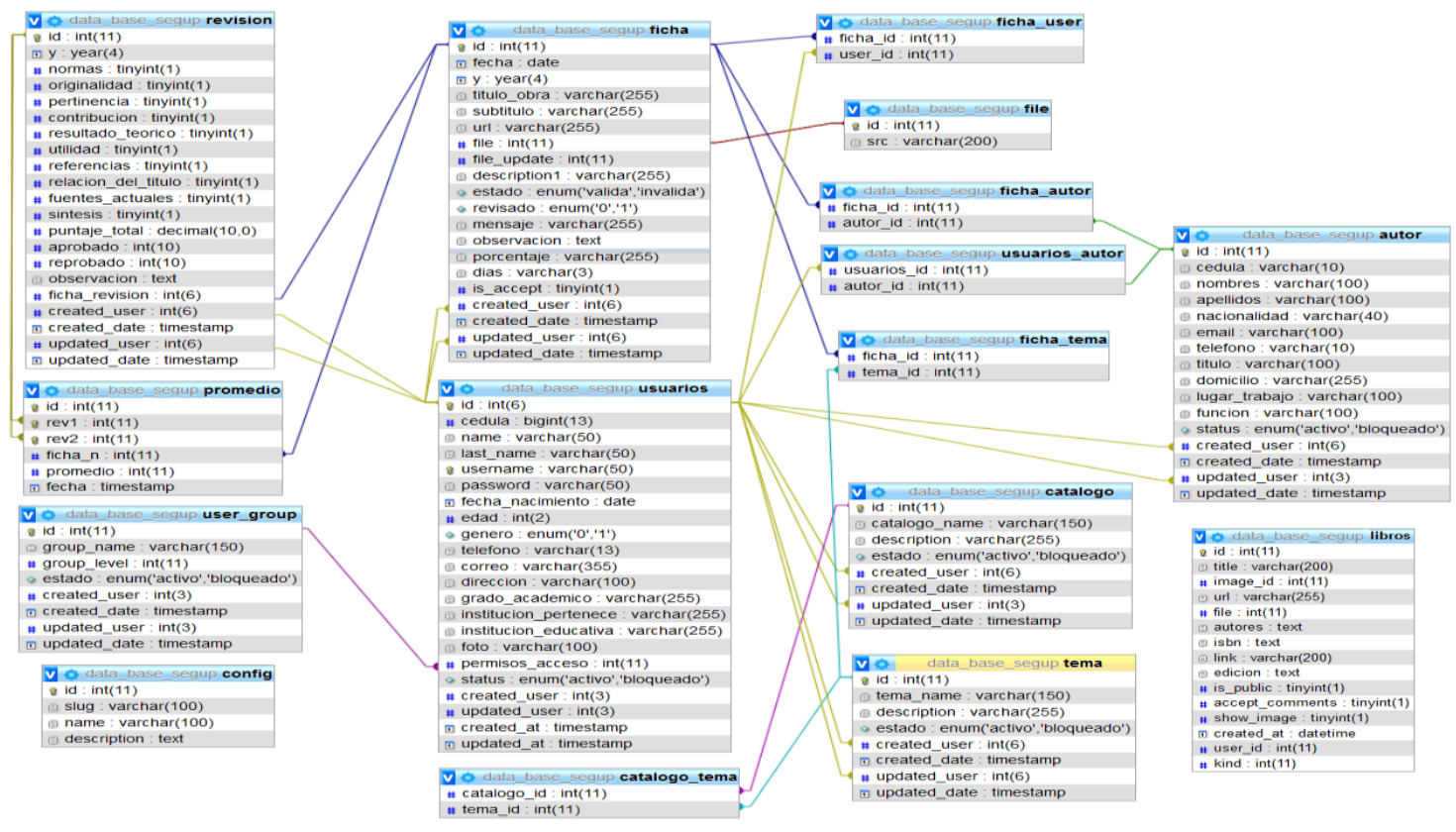

Figura 2. Base de datos del SEGUP

\subsection{Implementación de la plataforma}

La metodología Scrum tiene aún más relevancia, debido a que nos proporciona una serie de valores que, al momento de llevar a cabo el desarrollo del proyecto, ayudan a mantener un ambiente cordial entre los involucrados con el proyecto, y estos valores pueden ser:

- Compromiso con el desarrollo.

- Concentración en las metas.

- Franqueza entre involucrados.

- Respeto y coraje.
- Proactividad

con los involucrados.

\subsubsection{Fundamentación de la plataforma}

Se fundamenta el uso de la metodología de diseño de proyectos SCRUM para manejar los ciclos de desarrollo interactivo e incremental:

- Sistema modular: se pueden presentar diversas etapas en el proceso de diseño y desarrollo, debido a las características fundamentales del Sistema Editorial de Gestión Universitaria 
de Publicaciones de la Editorial Uleam, las cuales se verán en la necesidad de implementar con el complemento de las funcionalidades correspondientes y edición en el funcionamiento del sistema, determinando en cada aumento un producto con funciones 0 apariencia nuevas a las ya establecidas.

- Presentaciones regulares de las funciones desarrolladas a los actores principales de la editorial, para poder brindar nuevas versiones del sistema web y de esta forma realizar mejoras continuas en cortos períodos de tiempo.
- Predecible en cuanto a inestabilidades se refiere: i) incremento de funciones detectadas en las primeras etapas del proyecto. ii) Cambios repentinos que pueden surgir durante la ejecución del proyecto en lo que respecta a los requerimientos de usuarios 0 a los módulos desarrollados.

\subsection{Registro del sistema}

En la figura 3, se presenta el diagrama que permite detallar la interacción que tiene el usuario con el sistema, desde que el usuario se registra hasta el proceso de solicitud de una revisión.

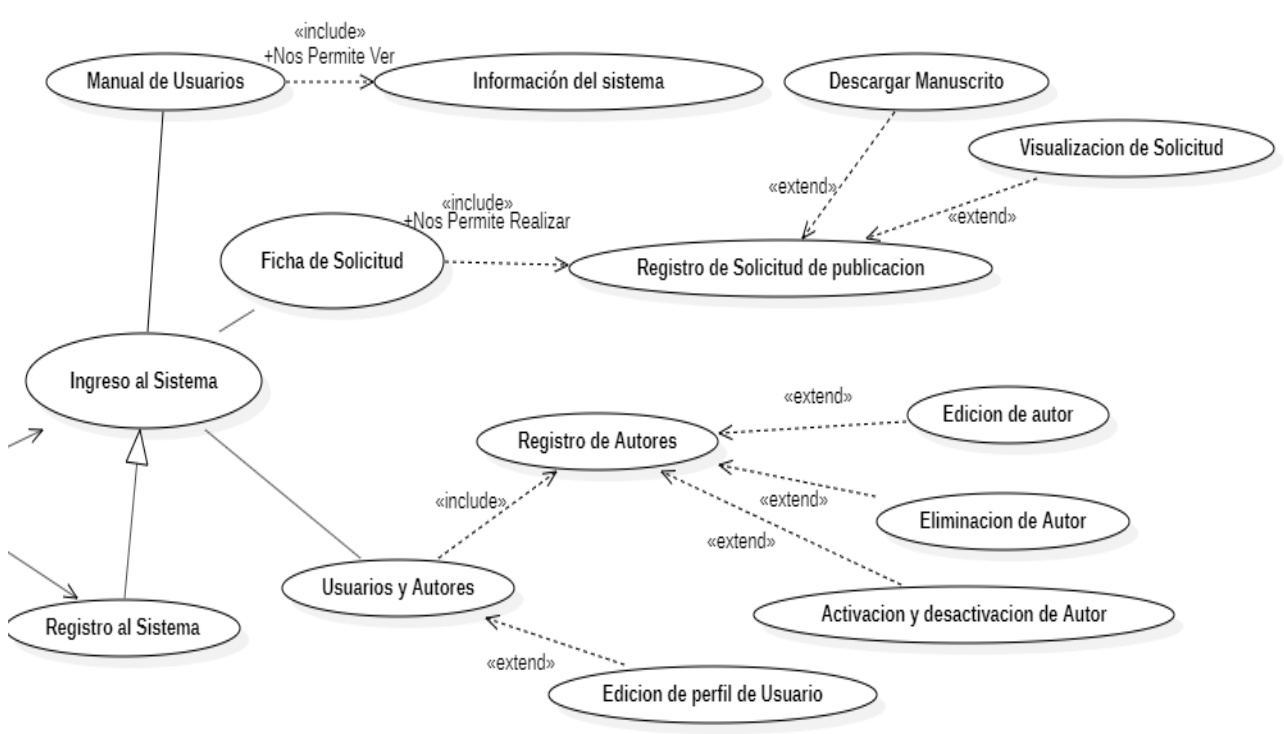

Figura 3. Diagrama de casos-usuarios-administrador del SEGUP 


\subsection{Especificación de diagrama} de actividad

El siguiente diagrama de actividad se emplea para poder observar de forma general las actividades principales que el sistema cumple. La figura 4 muestra el flujo asociado con las actividades desarrolladas en el proceso editorial.

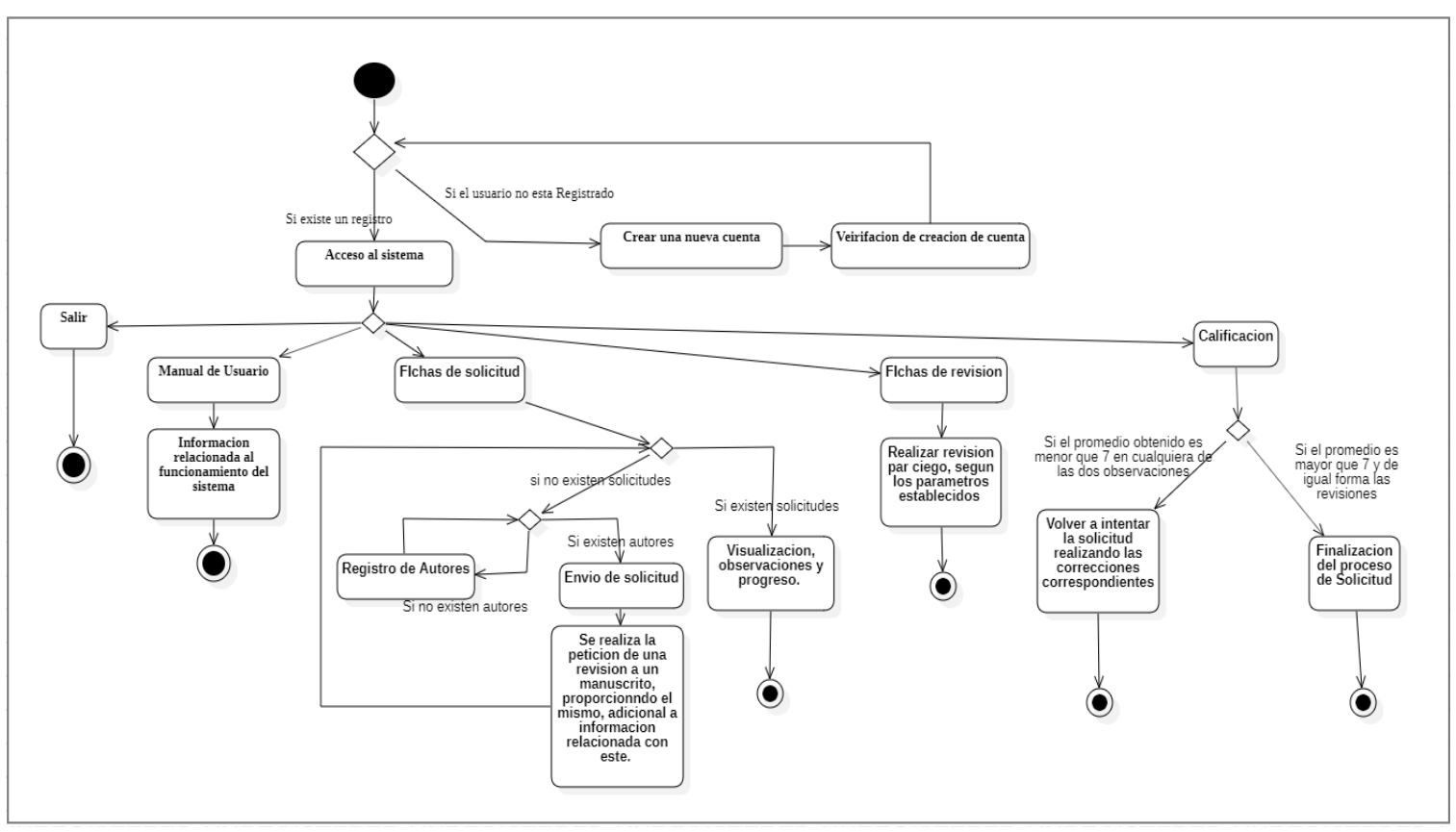

Figura 4. Diagrama de actividades-SEGUP

\subsection{Listado de características por funcionalidad}

\subsubsection{Editorial Universitaria}

- Gestión de contenido científico cargado en la plataforma.

- Verificación, calificación y aprobación de textos a difundir.

- Revisión y corrección de errores en los manuscritos.

- Gestión de usuarios (revisores y editores).

- Gestión de catálogos y temas.

- Administración del sistema.

\subsubsection{Usuarios}

- Solicitud de publicación.

- Carga de manuscritos sometidos a publicación.

- Asignación de colaboradores.

- Generación de múltiples solicitudes.

\subsubsection{Ambos}

- Administración de perfiles.

- Registro, inicio de sesión y cierre de sesión. 


\subsection{Registro de propiedad}

Una vez terminado el desarrollo del sistema se realizó el proceso de registro de propiedad intelectual del presente trabajo, en la dirección nacional de derechos de autor y derechos conexos con número de certificado: GYE-010068 para el posterior respaldo de lo desarrollado en este trabajo.

\section{Conclusiones}

La información requerida fue proporcionada por los colaboradores de la Editorial Universitaria, brindando facilidades en el levantamiento de los requerimientos, cabe señalar que estos sirvieron como puntos de partida para el diseño, desarrollo e implementación del sistema.

Se elaboró el diseño de la estructura del sistema web utilizando Visual Code como editor de código, xampp para el levantamiento del servidor virtual y la base de datos MySQL para el levantamiento de la conexión, para así posteriormente desarrollar las respectivas funcionalidades. Estas funcionalidades y módulos fueron posibles gracias a la utilización de la metodología SCRUM, debido a que la misma proporciona métodos que ayudan a cumplir con los tiempos que fueron establecidos, según la planificación descrita en este trabajo.

Se llevó a cabo la implementación del sistema editorial de gestión universitaria de publicaciones en la editorial de la Uleam, dando como resultado una acogida positiva por parte de la comunidad que interactúa con estos procesos.

\section{Bibliografía}

Artola-Valle, J. C., \& Gaitán-Orozco, M. C. (2016). Desarrollo e implementación del portal de la revista Científica "RCIEN" de la facultad de Ciencias e Ingenierías de la UNANManagua (Doctoral dissertation, Universidad Nacional Autónoma de Nicaragua).

Diego, G. J. (2012). El gran libro de HTML, CSS3 y JavaScript. $M A R C O M B O, S A$. Primera edición en libro electrónico: Enero de 2012.

Fernández-Martínez, J. D., Cadavid, A. N., \& Morales-Vélez, J. (2013). Revisión de metodologías ágiles para el desarrollo de software.

Flanagan, D., (2007) JavaScript, la guía definitiva. Madrid: Anaya Multimedia.

Fumas, E., (2014). brugor.com. ¿Qué es PHP? ¿Para qué 
sirve? Recuperado el 12/09/2019

de https://www.ibrugor.com/blog/ que-es-php-para-que-sirve/

Gustavo, B. (2019). Hostinger. ¿Qué es Apache? Descripción completa del servidor web Apache. Recuperado el 14/09/2019 de https://www.hostinger.com.ar/ tutoriales/que-es-apache/

Jorge, A. (2016). basicinfoweb. Aplicaciones Informáticas. Recuperado el 12/09/2019 de https://basicinfoweb.wordpres s.com/2016/04/20/que-sonlos-servicios-web-y-para-quesirven/

Mamaui-Tola, A. R. (2013). Implementación del sistema de gestión de proyectos de investigación (G project). Revista Ventana Científica, 1,2 .

Microsoft. (2019). Visual Studio Code. Getting Started. Recuperado el 15/11/2019 de https://code.visualstudio.com/ docs
Mozilla. (2019a). MDN Web Docs. CSS. Recuperado el 11/09/2019 de https://developer.mozilla.org/ es/docs/Web/CSS

Mozilla. (2019b). MDN Web Docs. HTML5. Recuperado el 11/09/2019 de https://developer.mozilla.org/ es/docs/HTML/HTML5

Rouse, M., (2015). Techtarget. Guía Esencial: Las bases de datos dan soporte a las tendencias de TI. Recuperado el 13/09/2019 de https://searchdatacenter.techt arget.com/es/definicion/MyS QL

Zepeda, R. (2015). El informático.com. ¿que es XAMPP? Recuperado el 13/09/2019 de http://blogdelinformaticoreizer.blogspot.com/2015/11/ que-es-xampp.html 